\title{
Design of Intelligent Power Distribution Terminal Based on DSP
}

\section{Zu Zhang ${ }^{1}$, Qing Zhang ${ }^{1}$, Tongtong Sun ${ }^{1}$}

\author{
${ }^{1}$ College of Mechanical and Electrical Engineering, Agricultural University of Hebei, Baoding Hebei 071001
}

\begin{abstract}
In view of the traditional distribution terminal reactive power compensation effect is poor, the response is not quick enough and so on, this paper designs a kind of intelligent power distribution terminal device based on TMS320F28335 chip DSP. Reactive power compensation module using switching virtual circuit(SVC), proposed by improving the C.P.Steinmetz theory, we derive a containing power factor compensation admittance algorithm, reactive power theory and practical formula using instantaneous with this algorithm as SVC control strategy. The distribution terminal can realize dynamic reactive power compensation system, improve the three-phase unbalance,effectively prevent excessive compensation, but also be used for the on-line monitoring of distribution transformer has a rich communication interface can also be used for online monitoring of the transformer, has a rich communication interface. Simulation results show the algorithm has high response speed and accuracy.
\end{abstract}

Keywords: intelligent power distribution terminal; DSP; Static Var Compensator ; Reactive power compensation

\section{Introduction}

Distribution terminals as the distribution network automation system, the underlying equipment in the distribution network management system plays an important role $^{[1]}$. Power management as an important function of distribution terminals, is an essential part of its ${ }^{[2]}$ Static Var Compensator (SVC) is an important means of reactive power compensation in power system. It can not only be used for reactive power compensation of power supply network, but also can be used to improve the three-phase imbalance and suppress voltage flicker. SVC is to control the thyristor firing angle to change the value of the SVC access system to change the absorption or reactive power, so the SVC control method is the key to improve the system performance $\mathrm{e}^{[3 \sim 5]}$.

The control strategy for SVC has been studied by many scholars ${ }^{[6 \sim 11]}$. In [6], the SVC voltage control method based on the improved simplex algorithm for nonlinear PI controller is proposed, which realizes the compensation of reactive power and three-phase imbalance. [7] proposed a variable structure based on neuron tuning PID var compensator voltage control strategy, effectively shortening the adjustment time, reduce the overshoot. In [8], a SVC adaptive backstepping passive feedback voltage control method is studied to improve the performance of SVC. In [9-11], nonlinear SVC control methods based on genetic algorithm and fuzzy algorithm are studied. However, these algorithms are complex and computationally expensive, which are not easy to implement.

Based on the theory of C.P.Steinmetz, this paper improves the compensation conditions in Steinmetz theory, and then deduces the practical SVC compensation formula using instantaneous reactive power theory. On this basis, TMS320F28335 DSP chip is adopted as the core device of control. The real power, reactive power and power factor are monitored in real time by monitoring the basic physical quantity of three-phase voltage and current. Through comprehensive analysis and processing of the data, Three-phase balance, to achieve the purpose of the specified power factor, and to prevent over-compensation occurred.

\section{SVC control strategy}

Distribution terminal is one of the main work is based on the three-phase voltage transformer and three-phase current to calculate the thyristor control angle $\alpha$, with $\alpha$ to change the current TCR. That is, by changing the current in TCR to adjust the reactive power. To know the control angle $\alpha$, we must first calculate the compensation charge.

This article is published under the terms of the Creative Commons Attribution License 4.0

Author(s) retain the copyright of this article. Publication rights with Alkhaer Publications.

Published at: http://www.ijsciences.com/pub/issue/2017-02/

DOI: 10.18483/ijSci.1183; Online ISSN: 2305-3925; Print ISSN: 2410-4477 


\subsection{Power Factor Adjustable C.P.Steinmetz Algorithm}

In the balance of three-phase load at the same time, the power factor can be fully upgraded to 1 to achieve compensation ${ }^{[12]}$. If the full compensation for the control target, due to signal acquisition, processing and transmission errors will easily be compensated for the electrical equipment constitutes a security risk. Therefore, both from the consideration of reducing line losses or from over-compensation to consider, the power factor is not necessary to compensate to 1. By improving the principle of C.P.Steinmetz three-phase unbalanced load compensation, the power factor is added to the compensation admittance formula, which not only can achieve the best economic benefit, but also can effectively suppress the overcompensation and realize the accurate tracking of the power factor.

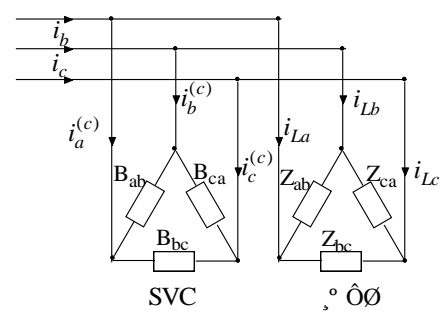

Fig.1 Equivalent circuit of SVC

According to Fig.1, the load fundamental wave current is decomposed into positive sequence component and negative sequence component according to the symmetrical component method, expressed by instantaneous value.

Positive sequence component :

$$
\left\{\begin{array}{l}
i_{L a+}=\sqrt{2} I_{L+} \cos \left(\omega t+\varphi_{L+}\right) \\
i_{L b+}=\sqrt{2} I_{L+} \cos \left(\omega t-\frac{2 \pi}{3}+\varphi_{L+}\right) \\
i_{L b+}=\sqrt{2} I_{L+} \cos \left(\omega t+\frac{2 \pi}{3}+\varphi_{L+}\right)
\end{array}\right.
$$

Negative sequence component :

$$
\left\{\begin{array}{l}
i_{L a-}=\sqrt{2} I_{L^{-}} \cos \left(\omega t+\varphi_{L-}\right) \\
i_{L b-}=\sqrt{2} I_{L^{-}} \cos \left(\omega t+\frac{2 \pi}{3}+\varphi_{L^{-}}\right) \\
i_{L b-}=\sqrt{2} I_{L^{-}} \cos \left(\omega t-\frac{2 \pi}{3}+\varphi_{L^{-}}\right)
\end{array}\right.
$$

Where, $I_{L+} 、 I_{L_{-}} 、 \varphi_{L^{+}} 、 \varphi_{L_{-}}$respectively, the load fundamental current positive sequence component, negative sequence component effective value and initial phase angle. The symmetrical component method is also used to divide SVC compensation current fundamental wave into positive sequence component: $i_{a+}^{(c)}$ 、 $i_{b+}^{(c)} 、 i_{c+}^{(c)}$, negative sequence component $i_{a-}^{(c)}$ 、 $i_{b-}^{(c)} 、 i_{c-}^{(c)}$. If the power factor of the equivalent load after compensation is $\lambda$, the positive-sequence component of the compensated a-phase base-line fundamental current should be:

$$
i_{a+}=\sqrt{2} I_{a+} \cos \left(\omega t-\varphi_{a+}\right) \quad \text { (3) . }
$$

where $\lambda=\cos \varphi_{a+}, I_{a+}$ is the effective value of the positive-sequence component of the bus-bar fundamental current. From (1) and (3) can be compensated positive fundamental current component of the fundamental wave:

$$
\begin{aligned}
& \left\{\begin{array}{l}
i_{a+}^{(c)}=\sqrt{2} I_{L+}\left(k \cos \varphi_{L+}+\sin \varphi_{L+}\right) \sin (\omega t) \\
i_{b+}^{(c)}=\sqrt{2} I_{L+}\left(k \cos \varphi_{L+}+\sin \varphi_{L+}\right) \sin \left(\omega t-\frac{2 \pi}{3}\right) \\
i_{c+}^{(c)}=\sqrt{2} I_{L+}\left(k \cos \varphi_{L+}+\sin \varphi_{L+}\right) \sin \left(\omega t+\frac{2 \pi}{3}\right)
\end{array}\right. \\
& \left(k=\sqrt{1-\lambda^{2}} / \lambda\right)
\end{aligned}
$$

In order to achieve the goal of three-phase balance, the sum of the negative-sequence component of the load fundamental current and the negative-sequence component of the compensated fundamental current is zero. which is:

$$
\left\{\begin{array}{l}
i_{a-}^{(c)}+i_{L a-}=0 \\
i_{b-}^{(c)}+i_{L b-}=0 \\
i_{c-}^{(c)}+i_{L c-}=0
\end{array}\right.
$$

Finally, by superposition theorem, we can get the compensation of each phase of SVC:

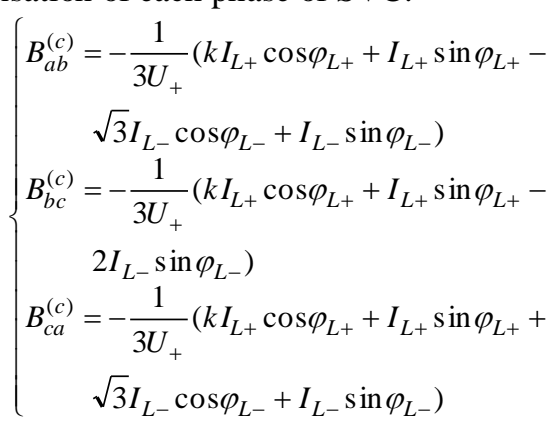

\subsection{Practical admittance compensation algorithm based on instantaneous reactive power theory}

For these components in (6), if we adopt the traditional vector theory to collect, we need to collect at least one cycle before we can calculate, making the SVC adjustment time is long, and the dynamic characteristic is bad. In this paper, the instantaneous reactive power theory of $i_{p} 、 i_{q}$ operation, the rapid derivation of the components, to obtain a practical compensation for the absorption of the formula.

The phase angle and angular velocity of the positive-sequence component of the positive-sequence component of the fundamental voltage of the positive-sequence component of the fundamental voltage of the bus are obtained. The three-phase load current signal is converted to the positive and negative synchronous rotating coordinate system using this as the reference.which is easy to be compensated for the required components of the charge. 
The positive sequence synchronous rotation of the load current transform, the fundamental sequence of its positive components see type (1), into the amount of direct current $I_{d+} 、 I_{q+}$, the other into the exchange of $I_{d+}^{\prime} 、 I_{q_{+}}^{\prime}$, which is:

$$
\left(\begin{array}{c}
I_{d+}+I_{d+}^{\prime} \\
I_{q_{+}}+I_{q+}^{\prime}
\end{array}\right)=\left(\begin{array}{c}
\sqrt{3} I_{L+} \cos \varphi_{L+}+I_{d+}^{\prime} \\
-\sqrt{3} I_{L+} \sin \varphi_{L+}+I_{q+}^{\prime}
\end{array}\right)
$$

Similarly, its fundamental negative sequence component see (2), into the direct flow of $I_{d-} 、 I_{q_{-}}$, the other into the exchange of $I_{d-}^{\prime}$ 、 $I_{q^{-}}^{\prime}$, which is:

$$
\left(\begin{array}{l}
I_{d-}+I_{d-}^{\prime} \\
I_{q_{-}}+I_{q^{-}}^{\prime}
\end{array}\right)=\left(\begin{array}{c}
\sqrt{3} I_{L_{-}} \cos \varphi_{L_{-}}+I_{d-}^{\prime} \\
-\sqrt{3} I_{L_{-}} \sin \varphi_{L_{-}}+I_{q^{-}}^{\prime}
\end{array}\right)
$$

After the synchronous rotation of the load current signal through the low-pass filter to get the amount of direct current, with people (6), we can get with the power factor of the practical compensation of the charge formula:

$$
\left\{\begin{array}{l}
B_{a b}^{(c)}=-\frac{\sqrt{3}}{9 U_{+}}\left(k I_{d+}-I_{q_{+}}-\sqrt{3} I_{d-}-I_{q_{-}}\right) \\
B_{b c}^{(c)}=-\frac{\sqrt{3}}{9 U_{+}}\left(k I_{d+}-I_{q_{+}}+2 I_{q_{-}}\right) \\
B_{c a}^{(c)}=-\frac{\sqrt{3}}{9 U_{+}}\left(k I_{d+}-I_{q+}+\sqrt{3} I_{d-}-I_{q-}\right)
\end{array}\right.
$$

\section{Hardware and Software Design of Intelligent Distribution Terminal}

Distribution terminal to DSP for the management and control center, with the SVC, transformer operating parameters to achieve real-time acquisition and local reactive power compensation. The overall structure of the system shown in Figure 2. Mainly by the main controller module, signal acquisition and conditioning module, communication module and reactive power compensation module and other modules.

After the terminal power-on, after a certain delay, and then work, through the distribution transformer three-phase voltage, three-phase current sampling, calculate the system's active power, reactive power and power factor,And compared with the user-set power factor range, then consider the system voltage amplitude to determine the static var compensation device switching, switching instruction sent to the trigger circuit, trigger SVC thyristor reactive power compensation. Not only can effectively improve the power quality, improve power factor, but also reduces the network loss.

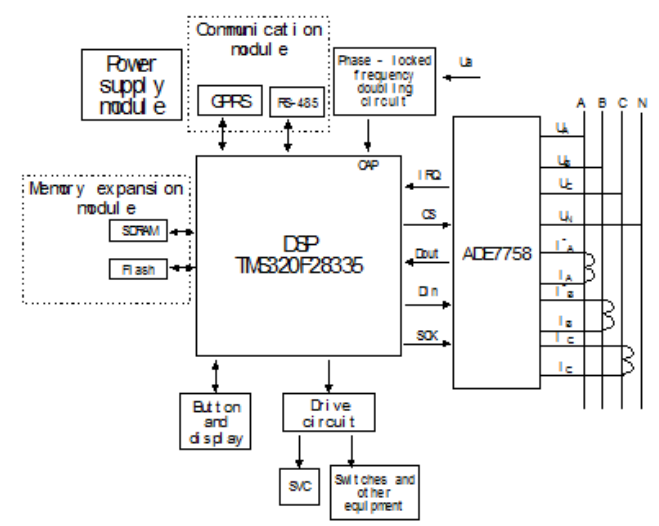

Fig.2 Intelligent power distribution terminal block diagram

\subsection{Power supply and reset circuit design}

DSP chip work when the voltage required for the I / O supply voltage $3.3 \mathrm{~V}$ and core voltage $1.9 \mathrm{~V}$. In this paper, the use of power supply chip TPS767D301 produce $3.3 \mathrm{~V}$ and $1.9 \mathrm{~V}$ voltage for DSP use. $0.1 \mu \mathrm{F}$ capacitor in the input terminal to filter out noise, improve response speed.

Chip TPS767D301 itself can generate a reset signal, and can supply the DSP to use, so do not set a special DSP reset chip. DSP and reset signal connection shown in Figure 3, pin 28, once the power on the power, the system is in a reset state, when $\overline{\mathrm{XRS}}$ is low, DSP reset.

\subsection{Design of phase-locking frequency multiplier circuit}

The power distribution terminal adopts the phase-locked frequency multiplication technique to generate the sampling pulse synchronously with the measured AC current signal so as to synchronize with the transformer signal. The start signal of the AD sampling adopts the high pulse train formed by the input signal frequency multiplication.

Phase-locked frequency multiplier circuit generally consists of zero-crossing trigger circuit, frequency multiplier circuit and phase-locked loop circuit. In this paper, CD4024 chip to achieve multiplier circuit, CD4046 chip to achieve phase-locked loop circuit, the two chip connection diagram shown in Figure 4. By CD4046 phase comparison within the circuit, voltage-controlled oscillator circuit through the circuit of the square wave signal phase locked; 128 times the frequency of the synchronization signal is implemented by CD4024, produced by pulse train input to the DSP started as AD synchronization using eCAP pin signal.

Because the phase-locked loop has the characteristics of real-time tracking, so when the detected signal changes, the circuit can automatically track, so you can ensure that each frequency cycle sampling interval 128 points. CD4046 and CD4024 connection shown in Figure 4. 


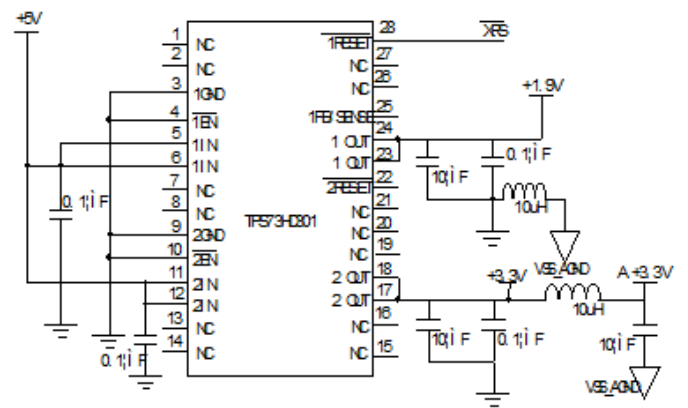

Fig.3 DSP Power Circuit

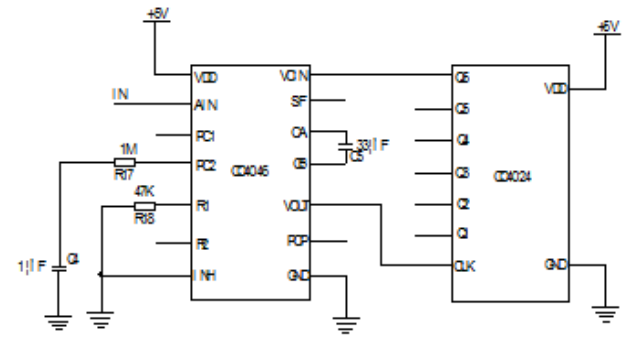

Fig.4 CD4046 and CD4024 connection diagram

\subsection{Software design}

System software uses a watchdog design, DSP running status monitoring to ensure the normal operation of the program to prevent system crashes. System program includes the main program, signal acquisition and processing procedures, such as reactive power compensation procedures. The main program is mainly used for system initialization and coordination between the various procedures. Signal acquisition and processing procedures for the completion of the read parameters and related operations.

According to the actual situation, pre-set power factor needs to reach the range. Read from the storage module, after system running continuously and the acquisition of distribution transformer parameters are got after computing power factor comparison, determine whether the need for reactive power compensation, if you need are in the process of reactive power compensation, according to the practical compensation susceptance formula, the formula (9), calculate the need compensation susceptance, again through the look-up table which is the control Angle $\alpha$.Issued by the DSP switching instructions, and thus control the TCR switching.

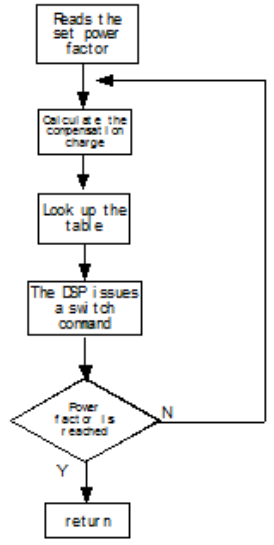

Fig.5 Reactive power compensation program

\section{Simulation verification}

In order to verify the accuracy of the algorithm, using Matlab simulation software to build three-phase three-wire imbalance load simulation model, the bus voltage: $10.5 \mathrm{kV}$, SVC compensation capacity: 120kvar. Set the TCR and load are used delta connection, because the TCR current is not connected non-sine wave, will produce harmonics, so add a capacitor (FC) to eliminate harmonics. Unbalanced load capacity table as shown in Table 1.

Tab.1 A set of unbalanced load capacities

\begin{tabular}{|c|c|c|c|}
\hline & A & B & C \\
\hline $\begin{array}{c}\text { Active power } \\
(\mathrm{kVA})\end{array}$ & 64 & 42 & 29 \\
\hline $\begin{array}{c}\text { Reactive } \\
\text { power } \\
(\mathrm{kW})\end{array}$ & 67 & 50 & 33 \\
\hline
\end{tabular}

The power factor after compensation is set to 0.9 and 0.95 . After the simulation, the SVC device is put into $0.1 \mathrm{~s}$, and the power values after compensation are shown in Table 2 . The simulation results show that the proposed algorithm can not only effectively balance the three-phase active power, achieve the specified power factor, but also has a high response speed. 
Tab.2 Compensation after each phase power

\begin{tabular}{|c|c|c|c|c|}
\hline & & $\mathrm{A}$ & B & $\mathrm{C}$ \\
\hline \multirow{2}{*}{$\lambda=0.9$} & $\begin{array}{l}\text { Active } \\
\text { power } \\
(\mathrm{kVA})\end{array}$ & 42.4 & 42.7 & 42.2 \\
\hline & $\begin{array}{c}\text { Reactive } \\
\text { power } \\
(\mathrm{kW})\end{array}$ & 20.0 & 19.9 & 20.3 \\
\hline \multirow[t]{2}{*}{$\lambda=0.95$} & $\begin{array}{l}\text { Active } \\
\text { power } \\
(\mathrm{kVA})\end{array}$ & 42.3 & 42.1 & 42.5 \\
\hline & $\begin{array}{c}\text { Reactive } \\
\text { power } \\
(\mathrm{kW})\end{array}$ & 13.9 & 13.5 & 13.8 \\
\hline \multicolumn{5}{|c|}{ Response time: $29 \mathrm{~ms}$} \\
\hline
\end{tabular}

\section{Conclusion}

In this paper, a high-performance intelligent power distribution terminal is designed. The terminal is DSP chip TMS320F28335, which is the core of management and control, and reactive power compensation is adopted FC+TCR static reactive power compensation device. For the three-phase unbalance, the modified SVC compensation algorithm based on instantaneous reactive power theory is deduced by improving the Steinmetz algorithm. The simulation results show that the proposed algorithm is accurate and rapid, and it can also prevent the reactive power from overcompensating. It also proves that the power distribution terminal can compensate the reactive power accurately and maintain the power factor to a good level and improve the power quality.

Using DSP design, a single CPU plus a small amount of external control circuit can form a complete distribution terminal. The system is simple and integrated, which improves the reliability and reduces the cost. It can be applied to the distribution transformer monitoring and dynamic reactive power compensation of low voltage system.

\section{REFERENCES:}

1) Liu Dong, Ding Zhenhua, et al. Key Technologies and Development of Distribution Automation Practicality. Automation of Electric Power Systems, 2004, 28 (7): 16-19.

2) Wang Ke, Zhang Zhanlong, Ma cuilian, et al. Online Monitoring System Terminal Design on Distribution Transformer. Proceedings of the CSU-EPSA, 2010, 22 (4): 56-59.

3) CONGA L, WANG Y, HILLD D J. Transient stability and voltage regulation enhancement via coordinated control of generator excitation and SVC[J].Electrical power and Energy Systems, 2005, 27(2): 121-130.

4) Zhu Jianjun. Reactive Power Compensation of Medium and Low Voltage Distribution. Power capacitor \& Reactive power Compensation, 2011, 32 (4): 19-23.

5) Yang Kun, Chen Lie, Chen Guozhu. Individual sequence control of DSTATCOM compensation for unbalanced load. Electric Power Automation Equipment, 2012, 32 (7): 36-41.

6) $\mathrm{Xu}$ Xianyong, Luo An, Fang Lu, et al. New Optimal Nonlinear PI Voltage Controller for SVC. Proceedings of the CSEE, 2009, 29 (1): 80-86.

7) Zhou Xiaohua, Wang Qi, Wang Zhifang, et al. Neuron variable structural PID control strategy for SVC. Electrical Measurement \& lnstrumentation, 2015, 52 (10): 61-65.

8) Zhang Lie, Zhang Aimin, Han Jiuqiang, et al. Adaptive backstepping passivity feedback control design for static volt-ampere reactive compensator. Control Theory \& Applications, 2012, 29 (3): 298-304.

9) Xu Yonghai, Xiao Xiangning, Yang Yihan, et al. POWER QUALITY DISTURBANCE IDENTIFICATION USIN G $\mathrm{dg}$ CONVERSION-BACFh NEURAL CLASSIFIER. Automation of Electric Power Systems, 2001，25 (14): 24-28.

10) Ding Qingqing, Wang Sanji. A NEW DESIGN OF TCR-TSC SVC CONTROLLER BASED ON NON-AFFINED NONLINEAR MODEL. Antomation of Electric Power Systems, 2002, 26 (14): 12-19.

11) Niu Wei, Fang Dazhong. Coordination Design of PSS and SVC Damping Controller Using GATS Hybrid Algorithm. Proceedings of the CSU - EPSA, 2006, 18 (1): 43-48.

12) AKAGI H, KANAZAWA Y, NABAEA.Generalised Theory of the Instantaneous Reactive Power in Three-phase Circuits[A].In : IEEE \& JIEE.Proceedings IPEC[C]. $1983: 1375$ - 1386. 